\title{
Prokaryotic silencing (psi)RNAs in Pyrococcus furiosus
}

\author{
CARYN HALE, ${ }^{1}$ KYLE KLEPPE, $^{1}$ REBECCA M. TERNS, $^{1}$ and MICHAEL P. TERNS ${ }^{1,2}$ \\ ${ }^{1}$ Departments of Biochemistry and Molecular Biology, University of Georgia, Athens, Georgia 30602, USA \\ ${ }^{2}$ Department of Genetics, University of Georgia, Athens, Georgia 30602, USA
}

\begin{abstract}
In many prokaryotes, noncoding RNAs that arise from the clustered regularly interspaced short palindromic repeat (CRISPR) loci are now thought to mediate defense against viruses and other molecular invaders by an RNAi-like pathway. CRISPR loci contain multiple short regions of similarity to invader sequences separated by short repeat sequences, and are associated with resistance to infection by corresponding viruses. It is hypothesized that RNAs derived from these regions, termed prokaryotic silencing (psi)RNAs, guide Slicer-like complexes of partner proteins to destroy invader nucleic acids. Here we have investigated CRISPR-derived RNAs in the archaeon Pyrococcus furiosus. Northern analysis revealed multiple RNA species consistent with a proposed biogenesis pathway that includes full-length CRISPR locus transcripts and intermediates generated by endonucleolytic cleavages within the repeat sequences. However, our results identify the principal products of the CRISPR loci as small psiRNAs comprised primarily of invader-targeting sequence with perhaps only 5-10 nucleotides of CRISPR repeat sequence. These RNAs are the most abundant CRISPR RNA species in $P$. furiosus and are likely the guides for the effector complexes of the proposed prokaryotic RNAi (pRNAi) system. We analyzed cell-free extracts fractionated under non-denaturing conditions and found that the various CRISPR RNA species are components of distinct RNA-protein complexes, including at least two complexes that contain mature-length psiRNAs. Finally, RNAs are produced from all seven CRISPR loci present in the $P$. furiosus genome, and interestingly, the most recently acquired psiRNAs encoded proximal to the leader sequence of a CRISPR locus appear to be the most abundant.
\end{abstract}

Keywords: CRISPR; prokaryotic RNAi; genome defense; viral defense; prokaryotic silencing (psi)RNAs

\section{INTRODUCTION}

Small, noncoding (nc)RNAs are found in all domains of life and function in a wide array of essential cellular processes. In eukaryotes, small ncRNAs, including siRNAs and microRNAs, have been shown to function in post-transcriptional gene silencing by targeting exogenous or endogenous RNAs, respectively, in a process called RNA interference, or RNAi (Hannon 2002). Another class of small RNAs referred to as piwi-associated RNAs (piRNAs) or repeat associated small interfering RNAs (rasiRNAs) regulates spreading of selfish genetic elements such as transposons or repeat elements in organisms including mammals, plants, and flies (Kim 2006; Nishida and Siomi 2006; Aravin et al. 2007; Hartig et al. 2007; Lin 2007).

Reprint requests to: Rebecca M. Terns, Department of Biochemistry and Molecular Biology, University of Georgia, Athens, GA, 30605, USA; e-mail: rterns@bmb.uga.edu; fax: (706) 542-1752; or Michael P. Terns, Department of Biochemistry and Molecular Biology, University of Georgia, Athens, GA, 30605, USA; e-mail: mterns@bmb.uga.edu; fax: (706) 542-1752.

Article published online ahead of print. Article and publication date are at http://www.rnajournal.org/cgi/doi/10.1261/rna.1246808.
An RNAi-like system that functions in genome defense has recently been proposed to exist in prokaryotes (Makarova et al. 2006; Deveau et al. 2008; Sorek et al. 2008; Tyson and Banfield 2008). The hallmark of the proposed prokaryotic RNAi (or pRNAi) system is the CRISPR locus, a cluster of short direct repeats that separate short variable sequences (i.e., clustered regularly interspaced short palindromic repeat). A number of the variable sequences (also sometimes called "spacers") found in CRISPR loci display complementarity (or identity) to known prokaryotic viruses, plasmids, and transposons (Bolotin et al. 2005; Mojica et al. 2005; Pourcel et al. 2005; Lillestol et al. 2006; Makarova et al. 2006). The other signature component of the hypothesized pRNAi system is a set of protein-coding genes referred to as CRISPR-associated or cas genes that are found in CRISPR-containing genomes (Jansen et al. 2002; Makarova et al. 2002, 2006; Haft et al. 2005). The cas genes are predicted to encode nucleases, helicases, RNA-binding proteins, and a polymerase (Jansen et al. 2002; Makarova et al. 2002, 2006; Haft et al. 2005). These bioinformatically predicted properties of the CRISPR and cas gene products led to the hypothesis that they comprise an RNAi-like system of genome defense in prokaryotes, in which RNAs 
derived from the variable regions of CRISPR loci (prokaryotic silencing or psiRNAs) guide the degradation of genome invaders by Cas proteins (Bolotin et al. 2005; Lillestol et al. 2006; Makarova et al. 2006). The Cas proteins are also expected to function in the processing of the psiRNAs and in the integration of new psiRNA genes (directed against newly encountered pathogens) into the genome.

Recent studies have provided strong evidence for a role of CRISPR loci in viral resistance in prokaryotes. Several groups have observed that virus exposure leads to the appearance of new virus-derived sequence elements within the CRISPR loci of surviving (resistant) isolates (Barrangou et al. 2007; Deveau et al. 2008; Horvath et al. 2008). In addition, Barrangou et al. (2007) showed that an alteration of an organism's CRISPR sequences that generate or destroy correspondence with a viral sequence results in viral resistance and viral sensitivity, respectively. However, the pathway by which CRISPR loci confer viral resistance remains hypothetical and undefined.

CRISPR loci are present in about half of bacterial genomes and nearly all archaeal genomes (Godde and Bickerton 2006; Makarova et al. 2006). A given locus can contain as few as two, and as many as several hundred, repeat-psiRNA units (Grissa et al. 2007; Sorek et al. 2008). The repeat sequences are generally $25-45$ nucleotides (nt) long and often weakly palindromic at the $5^{\prime}$ and $3^{\prime}$ termini (Jansen et al. 2002). Interspersed between the repeats are the variable, putative psiRNA-encoding sequences, which are usually similar in length to the repeats. RNAs arising from CRISPR loci have been detected by RNA cloning and/ or Northern blotting in three archaeal species: Archaeoglobus fulgidus, Sulfolobus solfataricus, and Sulfolobus acidocaldarius (Tang et al. 2002, 2005; Lillestol et al. 2006). These studies provided convincing evidence of transcription of entire CRISPR loci from the predicted transcriptional leader sequences that are found at one end of the loci, and of a discrete series of smaller RNAs that correspond in length to multiples of repeat-psiRNA units (e.g., $\sim 70$, $140,210,280 \mathrm{nt}$, etc.) (Tang et al. 2002, 2005). These findings along with RNA sequence analysis led to a hypothesized biogenesis pathway in which primary CRISPR transcripts are endonucleolytically cleaved within repeat sequences to produce psiRNAs flanked by repeat sequence at both the $5^{\prime}$ and $3^{\prime}$ ends (Tang et al. 2002, 2005). Intriguingly, very diffuse Northern blot signals suggesting smaller RNAs were also detected in S. acidocaldarius (Lillestol et al. 2006).

Here we have investigated the RNAs expressed from the seven CRISPR loci in Pyrococcus furiosus. Our results support the previously proposed steps in CRISPR RNA biogenesis but extend the pathway to include, as the apparent end products of biogenesis, small psiRNAs that likely contain less than $10 \mathrm{nt}$ of repeat sequence. Additionally, fractionation of $P$. furiosus extract reveals the presence of at least four potential psiRNA-protein complexes (psiRNPs), each containing distinct classes of CRISPR RNA species. The mature psiRNAs identified here and their associated complexes are leading candidates for the primary agents in the proposed prokaryotic RNAi viral defense system.

\section{RESULTS}

\section{psiRNAs cloned from the seven CRISPR loci in $P$. furiosus}

The $P$. furiosus genome contains seven CRISPR loci, each encoding between 11 and 51 and together encoding 200 potential psiRNAs (Fig. 1; Grissa et al. 2007). To investigate whether psiRNAs are produced from the seven CRISPR loci, we isolated and cloned small RNAs (less than 50 nucleotides) from total $P$. furiosus RNA preparations. Among 872 small RNA clones sequenced, 144 (17.3\%) were derived from CRISPR loci. In addition, $42.2 \%$ corresponded to rRNA, $23.9 \%$ were derived from ORFs, and $12.4 \%$ were from sRNAs (snoRNA homologs). The remaining $4.2 \%$ of sequences were derived from tRNAs, transposons, Hhc RNAs (Klein et al. 2002), and intergenic sequences.

Most of the CRISPR clones consisted primarily of psiRNA (variable) sequence and included some flanking repeat sequence. The clones included 64 of the 200 potential P. furiosus psiRNAs and represented all seven CRISPR loci (Fig. 1). We have adopted a simple system of nomenclature for psiRNAs, in which the psiRNA is designated by a three-digit number. The first digit indicates

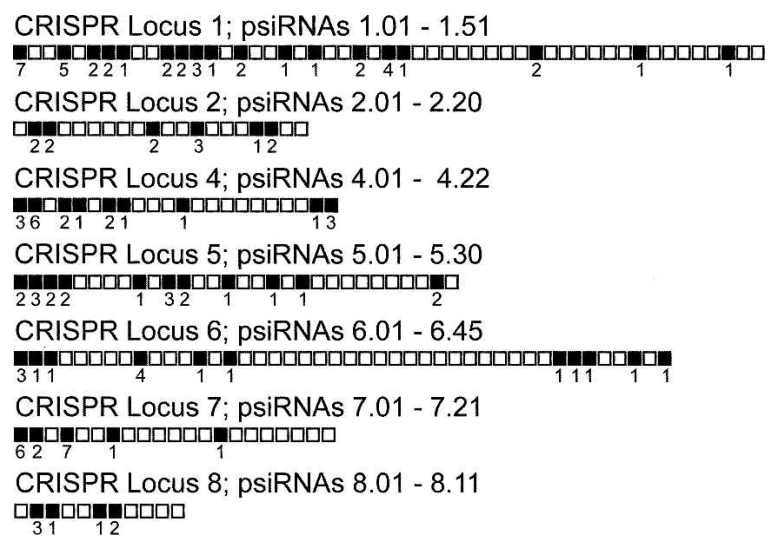

FIGURE 1. P. furiosus CRISPR loci and distribution of cloned psiRNAs. The seven $P$. furiosus CRISPR loci are illustrated. The psiRNA numbers associated with each locus are indicated above. Each psiRNA is represented by a box. Shaded psiRNAs were cloned at least once in this work, and the number of clones isolated is indicated below the shaded box. The genome coordinates of each $P$. furiosus CRISPR locus are as follows: 1, 27091-30618; 2, 260714-262113; 4, 312405-313931; 5, 623119-625176; 6, 695937-698992; 7, 10640761065543; and 8, 1091089-1091857. 
the locus number $(1,2$, and $4-8$ in P. furiosus), and the second two digits, separated from the first by a decimal point, indicate the position of the psiRNA within that locus (relative to the leader). For example, the first psiRNA in CRISPR locus 1 is Pf psiRNA 1.01, the first psiRNA in CRISPR locus 2 is psiRNA 2.01, and the last psiRNA in CRISPR locus 1 is psiRNA 1.51 (see Fig. 1). Figure 1 shows the number of times each individual psiRNA was cloned. The psiRNA clones ranged between 17 and $50 \mathrm{nt}$ in length (see Supplemental Table 1 for psiRNA clone sequences). The clones included variable amounts of the psiRNA sequence (12-40 nt) and of repeat sequence at the $5^{\prime}(0-8 \mathrm{nt})$ and/or 3' (0-22 nt) end. In addition to the psiRNA clones, we isolated a few CRISPR-derived clones that lacked psiRNA sequence and consisted of a portion of leader sequence upstream of a repeat (see "Leader" section of Supplemental Table 1), indicating that the 3 ' end of the CRISPR leader is also transcribed.

Our sampling is not apparently at saturation; however, we cloned psiRNAs from the beginning, middle, and end of CRISPR loci (Fig. 1), indicating that RNAs are produced from across the length of the loci. Interestingly, however, the likelihood of cloning was significantly higher for psiRNAs encoded within the first part of a CRISPR locus, suggesting a greater abundance in the organism of psiRNAs from these regions. Two-thirds of the psiRNAs that we cloned were from the first third of their CRISPR locus, and $45 \%$ were one of the first four psiRNAs in a given locus (Fig. 1). With the exception of locus 2, this trend was observed within each individual CRISPR locus.

Comparison of the percentage of psiRNAs cloned from a given locus (Table 1, percent of clones) to the percentage of the total psiRNAs encoded by that locus (Table 1, percent of psiRNAs) revealed that most of the loci are represented proportionately within the clones. However, locus 6 seems to be significantly underrepresented in the cDNA library. Locus 6 encodes $\sim 22 \%$ of the psiRNAs in P. furiosus; however, only $\sim 12 \%$ of the cloned RNAs were derived from this locus. This suggests that the psiRNAs encoded within locus 6 are less abundant in P. furiosus than those encoded by the six other CRISPR loci.

\begin{tabular}{lcccc}
\multicolumn{5}{l}{ TABLE 1. Distribution of cloned psiRNAs } \\
\hline $\begin{array}{l}\text { CRISPR } \\
\text { locus }\end{array}$ & $\begin{array}{c}\text { Number } \\
\text { of psiRNAs }\end{array}$ & $\begin{array}{c}\text { Number } \\
\text { of clones }\end{array}$ & $\begin{array}{c}\text { Percent } \\
\text { of psiRNA (\%) }\end{array}$ & $\begin{array}{c}\text { Percent } \\
\text { of clones (\%) }\end{array}$ \\
\hline 1 & 51 & 40 & 25 & 30 \\
2 & 20 & 12 & 10 & 9 \\
4 & 22 & 20 & 11 & 15 \\
5 & 30 & 20 & 15 & 15 \\
6 & 45 & 16 & 22 & 12 \\
7 & 21 & 17 & 10 & 13 \\
8 & 11 & 7 & 5 & 5 \\
Total & 200 & 132 & & \\
\hline
\end{tabular}

The results of the RNA cloning suggest the presence of novel, small psiRNAs in P. furiosus. However, the cloned RNAs were not of a uniform size or composition. To determine whether discrete psiRNA species are present in $P$. furiosus, we undertook additional analysis.

\section{Northern analysis of RNAs derived from the CRISPR loci in $P$. furiosus}

In order to further investigate the RNAs that arise from the CRISPR loci in P. furiosus, we undertook Northern analysis with probes against both repeat and psiRNA sequences. Probes were designed for detection of both sense (transcription from the leader sequence) and antisense RNAs. Figure 2A shows results obtained with a probe that recognizes the repeat sequence (sense orientation) that is common to $P$. furiosus CRISPR loci 1, 5, and 6. This probe detected a prominent band at $\sim 65 \mathrm{nt}$, a less prominent band at $\sim 130 \mathrm{nt}$, and an unresolved set of bands of greater than $150 \mathrm{nt}$ near the top of the gel. For this and all other probes tested, no significant differences in the patterns were observed from total RNA samples prepared with and without DNase treatment, indicating that the bands represent RNAs. Consistent with the observations and CRISPR RNA processing pathway proposed by others (Tang et al. 2002, 2005; Lillestol et al. 2006; Makarova et al. 2006; Sorek et al. 2008), the set of bands above $150 \mathrm{nt}$ likely represents a mixture of primary transcripts from the three loci as well as larger intermediates generated by cleavages within repeat regions. The most prominent band detected with the repeat probe in P. furiosus $(\sim 65 \mathrm{nt})$ corresponds well to the primary product of the CRISPR loci reported previously in other organisms (Tang et al. 2002, 2005; Lillestol et al. 2006); however, in this work this RNA is identified as the " $1 \mathrm{X}$ intermediate" (Fig. 2). This band corresponds in length to a psiRNA $(\sim 35-40 \mathrm{nt})$ and repeat $(\sim 30 \mathrm{nt})$ and likely represents psiRNAs with flanking repeat sequences generated by cleavages within the adjacent repeats (see Fig. $2 \mathrm{C}$ ). The detection of this RNA by the repeat probe suggests that cleavage may be asymmetric within the repeat sequence, leaving a substantial contiguous region of the 30-nt repeat on one side (e.g., the $3^{\prime}$ end as modeled in Figure 2C) for efficient detection by Northern probes. A less abundant band of $\sim 130 \mathrm{nt}$ corresponds in length to two psiRNAs with flanking repeat sequences and likely represents the immediately upstream $2 \mathrm{X}$ intermediates (Fig. 2C; Tang et al. 2002, 2005; Lillestol et al. 2006).

Northern analysis with a probe against one of the variable psiRNA sequences revealed novel CRISPR RNA species. Using a probe against psiRNA 4.02 (sense orientation), we detected a band at $\sim 60 \mathrm{nt}$ and a very faint signal near the top of the gel, but the most prominent band is $\sim 46 \mathrm{nt}$ (Fig. 2B) and corresponds in size to that of the psiRNA (35 nt in the case of psiRNA 4.02) and $\sim 10 \mathrm{nt}$ of repeat sequence. A significant secondary band was detected at $\sim 39$ nt (Fig. 2B). 

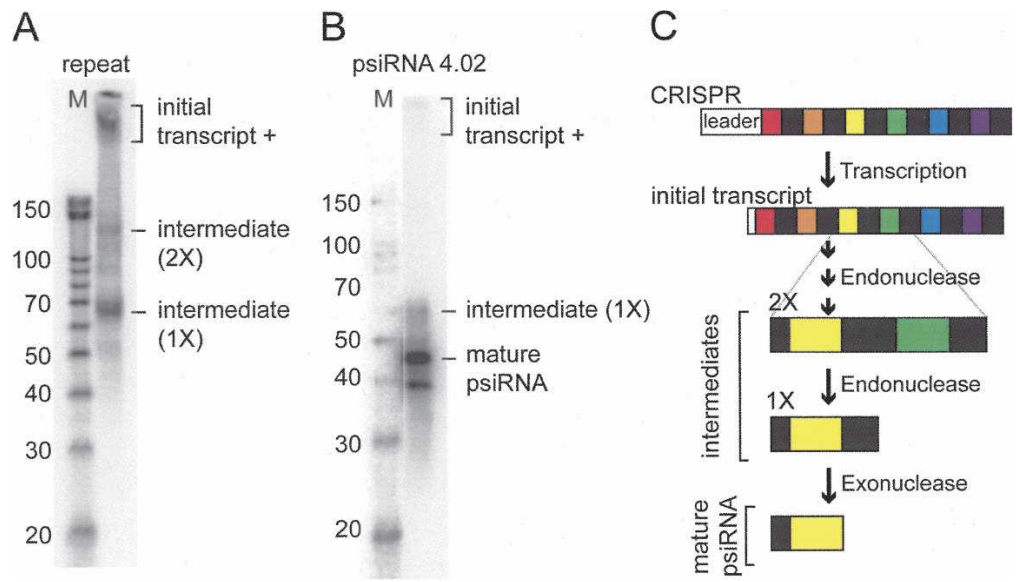

FIGURE 2. Northern analysis of RNAs containing CRISPR repeat and psiRNA 4.02 sequences. $(A, B)$ Northern blots were performed with $10 \mu \mathrm{g}$ total RNA using a degenerate oligonucleotide probe designed to detect the repeat sequences for CRISPR loci 1,5 , and $6(A)$ or a probe for psiRNA 4.02 sequence $(B)$. Radiolabeled RNA marker sizes are indicated $(M)$. The positions of the initial transcript and large intermediates, $2 \mathrm{X}$ intermediate, $1 \mathrm{X}$ intermediate, and primary mature psiRNA described in the text and Figure 4C are indicated. $(C)$ Proposed psiRNA biogenesis pathway. The CRISPR locus is transcribed from a start site within the leader sequence to produce an initial transcript that includes a portion of the leader and the alternating psiRNA and repeat sequences. The initial transcript is cleaved within the repeats to produce intermediates. The endonucleolytic cleavage site may be asymmetrically located within the repeat. The $2 \mathrm{X}$ intermediate and $1 \mathrm{X}$ intermediates are illustrated. Our results indicate that the $1 \mathrm{X}$ intermediate is further processed by an exonuclease to remove most of the repeat sequence, resulting in a primary mature psiRNA species that contains $5-10 \mathrm{nt}$ of the repeat sequence.

Importantly, similar results were observed in Northern analysis of RNAs from other CRISPR loci. Results for all RNAs analyzed, both sense and antisense, are compiled in Figure 3. First, like the repeat 1, 5, 6 probe, a probe for the repeat sequence common to loci 2, 4, and 7 (sense orientation) detected prominent diffuse bands of $\sim 65$ and $\sim 130 \mathrm{nt}$ (theoretical $1 \mathrm{X}$ and $2 \mathrm{X}$ processing intermediates) (see Fig. 2C). In addition, we probed for psiRNAs from the first part of each CRISPR locus (1.01, 2.01, 4.02, 5.02, 6.01, 7.01, and 8.01) as well as for psiRNA sequences from the middle and end of locus 7 (7.11 and 7.21). Strikingly, probes for each of the psiRNA sequences (sense orientation) detected a single predominant RNA species (indicated with dots in Fig. 3). Most of these predominant RNAs were $\sim 43$ to $\sim 46 \mathrm{nt}$. The observed size of the major RNA species was generally $5-10 \mathrm{nt}$ longer than the encoded psiRNA sequence. The psiRNA with the longest observed primary product (psiRNA 1.01) has an unusually long psiRNA sequence. These findings, together with the observation that these RNAs are recognized by psiRNA but not repeat sequence probes, suggest that the primary mature psiRNA species in $P$. furiosus consists of a psiRNA with $\sim 5-10$ nt of repeat.

In addition to the primary psiRNA species, each of the psiRNA probes detected other RNAs. These often included an RNA close to the size of the $\sim 65$-nt $1 \mathrm{X}$ intermediates that were detected by repeat probes, and in some cases (e.g., psiRNAs 101 and 402), an RNA was detected that was the size of the theoretical 2X intermediate. Many of the psiRNA probes detected other faint bands. However, in many cases the most prevalent secondary species was a slightly smaller RNA of $\sim 38$ to $\sim 45$ nt.

We did not detect antisense RNAs with most of the CRISPR probes (Fig. 3B). Prominent bands were detected with probes from psiRNAs 2.01 and 7.11; however, the absence of corresponding bands with the repeat probes suggests that these are not CRISPR locus-derived RNAs.

\section{Northern analysis of CRISPR RNA distribution in fractionated $P$. furiosus extract}

The CRISPR RNAs are hypothesized to function in complex with proteins in various aspects of RNA-guided genome defense in prokaryotes (Makarova et al. 2006). To assess whether the CRISPRderived RNA species that we identified may be components of complexes, we analyzed the distribution of the RNAs across fractions from anion exchange chromatography of $P$. furiosus S100 cell extract performed under anaerobic conditions. Fractions were evaluated by Northern analysis using probes against the repeat sequence common to loci 1,5 , and 6 , and psiRNA 4.02 and 7.01 sequences (Fig. 4). For reference, the profile of RNAs detected in unfractionated extract is shown in the first lane.

The distribution of the various RNAs across the fractions suggests the presence of several distinct CRISPR RNAcontaining complexes. The novel primary and secondary psiRNAs ( $\sim 45$ and $\sim 39$ nt) from both loci co-fractionated in a distinct set of fractions denoted as peak A (Fig. 4, fractions 10-14). Other larger CRISPR RNA species were not observed in peak A. The primary mature psiRNA is also present in peak B (Fig. 4, fractions 20-23) along with a fraction of the $1 \mathrm{X}$ intermediate RNA and some of the variable-size psiRNA species. However, the highest concentration of the $1 \mathrm{X}$ intermediate is found in a distinct set of fractions that lack small psiRNAs, termed peak C (Fig. 4, fractions 23-26). The mixture of RNAs that likely includes full CRISPR locus transcripts and larger intermediates and the $2 \mathrm{X}$ intermediate are found primarily in peak $\mathrm{D}$ (Fig. 4, fractions 31-34). These results suggest the presence of multiple complexes each containing distinct subsets of CRISPR-derived RNAs in P. furiosus.

To verify the presence of CRISPR RNA-protein complexes in the fractionated $P$. furiosus extract, we examined the complexes on native gels. Using the probe for psiRNA 7.01, we compared the mobility of the RNAs, both in the 
A

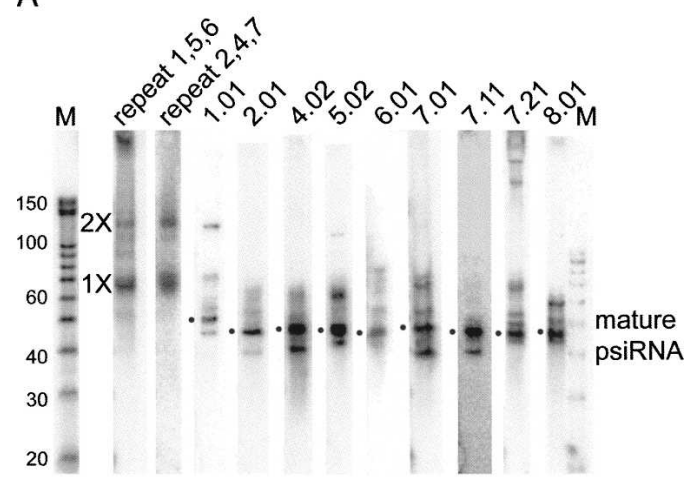

B

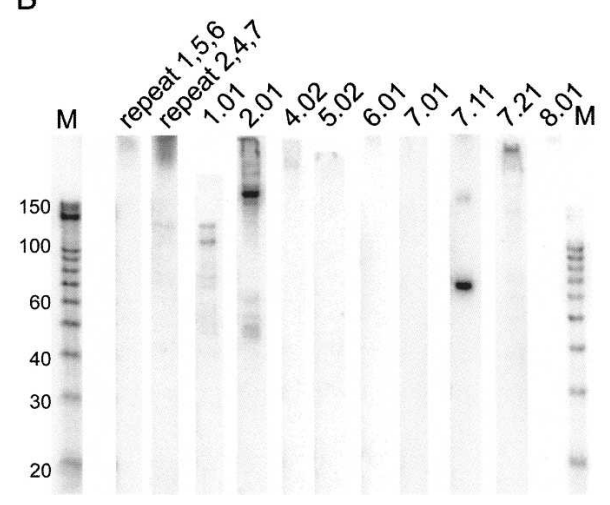

FIGURE 3. Northern analysis of RNAs from the seven $P$. furiosus CRISPR loci. $(A, B)$ Northern analysis was performed with $10 \mu \mathrm{g}$ of total RNA using probes to detect sense ( $A$; transcribed from the leader sequence) or antisense (B) psiRNA or repeat sequence-containing RNAs as indicated. Lanes are approximately aligned on the basis of adjacent marker lanes (not shown except for repeat 1, 5, 6 and psiRNA 8.01 lanes). Dots located to the left of lanes indicate the primary mature psiRNA species. $1 \mathrm{X}$ and $2 \mathrm{X}$ intermediates detected by repeat probes are indicated.

presence of the cofractionating proteins in peaks $\mathrm{A}, \mathrm{B}$, and $\mathrm{C}$ and following protein extraction, by non-denaturing PAGE and Northern analysis. In each case, a significant shift in the mobility of the RNAs was observed in the presence of the proteins. Peak D was not examined on native gels. Together our results indicate that the novel primary mature psiRNA is a component of at least two chromatographically distinct RNA-protein complexes (peaks $\mathrm{A}$ and $\mathrm{B}$ ), the $1 \mathrm{X}$ intermediate is found primarily in a third complex (peak C), and a fourth complex includes larger psiRNA precursors. These complexes are likely candidates for the mediators of psiRNA production, invader destruction, and CRISPR element integration in the proposed prokaryotic RNAi pathway.

\section{DISCUSSION}

\section{Novel CRISPR RNAs}

The CRISPR loci found in many prokaryotes encode alternating repeat and spacer or psiRNA sequences and have been shown to give rise to a series of RNAs that decrease in increments from the full-length locus transcript to a single psiRNA and repeat (i.e., $1 \mathrm{X}$ intermediate) (see Fig. 2C; Tang et al. 2002, 2005; Lillestol et al. 2006). Current evidence indicates that processing occurs by endonucleolytic cleavages within the repeat sequences (Tang et al. 2002, 2005). The RNA products of the CRISPR loci are hypothesized to guide silencing of viruses and other genome invaders. In this work, we have identified a novel class of smaller discrete CRISPR-derived RNAs that we have termed psiRNAs, which appear to be the ultimate gene products of the CRISPR loci (Fig. 2C).

The mature psiRNA species that we have identified is the most abundant CRISPR-derived RNA detected in $P$. furiosus. The primary mature psiRNAs are $\sim 5-10 \mathrm{nt}$ longer than the corresponding psiRNA sequence (i.e., $\sim 45$ nt long). These RNAs are shorter than the smallest

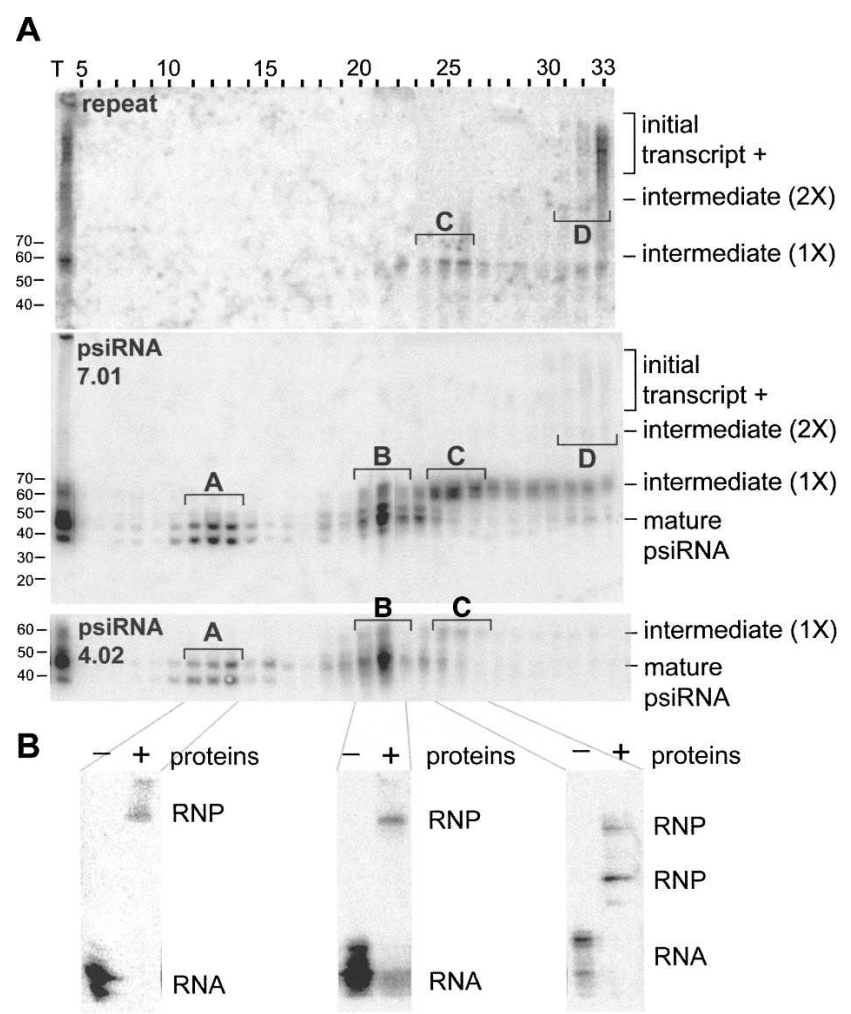

FIGURE 4. CRISPR RNA-protein complexes in fractionated $P$. furiosus cell extract. (A) P. furiosus S100 cell extract was separated by DEAE anion exchange chromatography and CRISPR RNAs present in the fractions were examined by Northern analysis using probes against repeat 1, 5, 6, and psiRNAs 7.01 and 4.02 as indicated. Unfractionated extract $(T)$ was coanalyzed for reference. Positions of $1 \mathrm{X}$ intermediate, $2 \mathrm{X}$ intermediate, and primary mature psiRNA, peaks A-D (see text), and markers are indicated. (B) Fractions corresponding to peaks A (left), B (center), and C (right) were analyzed by nondenaturing gel electrophoresis and Northern blotting using a probe against psiRNA 7.01. For comparison, proteins were extracted from a portion of each sample and analysis of the RNAs with $(+)$ and without $(-)$ proteins is shown. The positions of the RNAs (-proteins) and potential RNPs (+ proteins) on the native gel are indicated. 
discrete CRISPR RNA products previously reported (i.e., the $\sim 60$ - to 65 -nt $1 \mathrm{X}$ intermediate species) (Tang et al. 2002, 2005; Lillestol et al. 2006) and are presumably generated by exonucleolytic processing of the $1 \mathrm{X}$ intermediate. Our Northern and sequence analysis indicates that these RNAs are composed primarily of psiRNA sequence and do not contain substantial repeat sequence. A secondary psiRNA of $\sim 39 \mathrm{nt}$ was also consistently observed among psiRNA profiles.

\section{CRISPR RNA-protein complexes}

The common primary and secondary psiRNA species are likely candidates for the guide RNA component of the effector complex in the proposed pRNAi genome defense pathway. Both of these psiRNA species, but not larger intermediate CRISPR RNAs, are found in RNA-protein complexes in anion exchange chromatography peak A (Fig. $4 \mathrm{~A}, \mathrm{~B})$; thus, peak A could contain the effector complex. The primary mature psiRNA is even more abundant in peak $\mathrm{B}$, which contains relatively less of the secondary psiRNA but also contains some $1 \mathrm{X}$ intermediate RNA (Fig. 4). Peak B may also contain the effector complex and/or a complex involved in the exonucleolytic processing of the $1 \mathrm{X}$ intermediate to psiRNAs. The $1 \mathrm{X}$ intermediate RNA is most abundant in peak $\mathrm{C}$, which is adjacent to peak $\mathrm{B}$, and processing may occur across peak $\mathrm{B}$ and $\mathrm{C}$ fractions. Longer CRISPR RNAs are found in peak D. Our results indicate that the various RNA species are components of distinct RNA-protein complexes in P. furiosus. Extensive purification and analysis will determine whether these hypothesized activities and the Cas proteins predicted to function in CRISPR RNA biogenesis and invader silencing (e.g., RNA binding proteins and nucleases) are present in these complexes.

\section{psiRNA expression}

The psiRNAs are hypothesized to act in a manner similar to the antibodies of the human immune system, and expression would be expected even in the absence of active infection to patrol for returning invaders. Our results indicate that psiRNAs are actively produced from all seven CRISPR loci in P. furiosus. Moreover, expression levels appear to be equivalent between the loci under the growth conditions examined, with the possible exception of one locus that yielded 50\% fewer psiRNA clones than expected (see Table 1, locus 6). Our results confirm that CRISPR RNAs are transcribed from the leader sequence in $P$. furiosus and indicate that a portion of the leader sequence is also transcribed.

Interestingly, we found evidence of significantly higher levels of expression of psiRNAs encoded proximal to the leader of a CRISPR locus. Current data indicate that these are the most recently acquired psiRNA sequences within CRISPR loci (Pourcel et al. 2005; Barrangou et al. 2007). Increased levels of these psiRNAs may be important for targeting current invaders. Distal psiRNAs may be produced at lower levels to provide surveillance for past invaders. It is not clear whether the increased level of leader-proximal psiRNAs results from differences in RNA transcription (e.g., partial transcription of the loci), processing, stability, or other factors. This is an important new aspect of understanding the regulation of psiRNA expression that remains to be explored.

\section{MATERIALS AND METHODS}

\section{Small RNA cloning and sequencing}

Total RNA was isolated from P. furiosus cells using the Trizol reagent (Invitrogen) as indicated by the manufacturer. Approximately $300 \mu \mathrm{g}$ of total RNA was separated on an $8 \mathrm{~cm} \times 8.5 \mathrm{~cm}$ $15 \%$ polyacrylamide $7 \mathrm{M}$ urea gel using DNA size standards (pGEM markers, Promega) for size determination. RNAs between 20 and $50 \mathrm{nt}$ were isolated and passively eluted overnight in $0.5 \mathrm{M}$ ammonium acetate, $0.1 \%$ SDS, $0.5 \mathrm{mM}$ EDTA, followed by ethanol precipitation. In order to remove potential 5 ' triphosphates or cap structures, RNAs were treated with $50 \mathrm{U}$ tobacco acid pyrophosphatase (TAP) (Epicentre) for $2 \mathrm{~h}$ at $37^{\circ} \mathrm{C}$. The eluted RNAs were cloned using standard microRNA cloning protocols (Lau et al. 2001) using the following primers containing EcoRI restriction sites: $3^{\prime}$ adapter, $5^{\prime}$-AppTTTAACCGCGAATTC CAGddC-3' (IDT); 5' adapter, 5'-ACGGAATTCCTCACTrArAr A-3' (IDT); RT/PCR primer, 5'-GACTAGCTGGAATTCGCGGT TAAA-3' (IDT); and PCR primer, 5'-CAGCCAACGGAATTCCT CACTAAA-3'. An additional PCR was performed in order to add a BanI restriction site (GGYRCC) for concatamerization of the PCR products using the following primers: PCR2 5' primer 5'-GA CTAGCTTGGTGCCGAATTCGCGGTTAAA-3', PCR2 3' primer 5'-GAGCCAACAGGCACCGAATTCCTCACTAAA-3'. The products were subject to restriction digestion and DNA ligation by standard methods (Lau et al. 2001). cDNAs were cloned into the pCRII TOPO vector (Invitrogen) and transformed into TOP10 cells (Invitrogen) as described by the manufacturer. Plasmid preparation and sequencing was performed in a 96-well plate format using standard M13 forward, reverse, and T7 promoter primers. Sequences were analyzed using BLAST (NCBI).

\section{Northern analysis}

Approximately $10 \mu \mathrm{g}$ of $P$. furiosus total RNA was separated on $15 \%$ polyacrylamide $7 \mathrm{M}$ urea gels (Criterion, Bio-Rad) alongside $\left[{ }^{32} \mathrm{P}\right]-5^{\prime}$-end radiolabeled RNA markers (Decade, Ambion). The RNAs were transferred onto nylon membranes (Zeta-Probe, BioRad) using a Trans-Blot SD Semi-Dry Cell (Bio-Rad). Membranes were baked at $80^{\circ} \mathrm{C}$ for at least an hour before prehybridization in a ProBlot hybrdization oven (LabNet) for at least $1 \mathrm{~h}$ at $42^{\circ} \mathrm{C}$. Prehybridization and hybridization was performed in either Oligo-UltraHyb (Ambion) buffer or hybridization buffer contain-

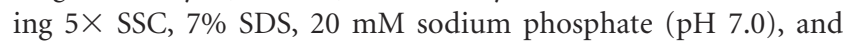
$1 \times$ Denhardt's solution. Deoxyribonucleotide probes (MWG) 
TABLE 2. Probes used in Northern analysis

Probe

Repeat 1,5,6 (antisense)

Repeat 1,5,6 (sense)

Repeat 2,4,7 (antisense)

Repeat 2,4,7 (sense)

psiRNA 1.01 (antisense)

psiRNA 1.01 (sense)

psiRNA 2.01 (antisense)

psiRNA 2.01 (sense)

psiRNA 4.02 (antisense)

psiRNA 4.02 (sense)

psiRNA 5.02 (antisense)

psiRNA 5.02 (sense)

psiRNA 6.01 (antisense)

psiRNA 6.01 (sense)

psiRNA 7.01 (antisense)

psiRNA 7.01 (sense)

psiRNA 7.11 (antisense)

psiRNA 7.11 (sense)

psiRNA 7.21 (antisense)

psiRNA 7.21 (sense)

psiRNA 8.01 (antisense)

psiRNA 8.01 (sense)
Sequence

CTTTCAATTCTATTTT(AG)GTCTTATTC(GT)AAC

GTT(AC)CAATAAGAC(TC)AAAATAGAATTGAAAG

CTTTCAATTCTTTTTGTAGTCTTATTGGAAC

GTTCCAATAAGACTACAAAAGAATTGAAAG

GGTCAGATCAGATTGCTTAAGACAAGAAATG

CATTTCTTGTCTTAAGCAATCTGATCTGACC

GTGGAGCAGAGTCAGAAGAAGAAGTGCG

CGCACTTCТTCTTCTGACTCTGCTCСAC

TCTGATAGGCTTCAAAGAGTGGCGCTTCAAC

GTTGAAGCGCCACTCTTTGAAGCCTATCAGA

GGGAATGGTTCACGTAGTACTTGAGGGCGC

GCGCCCTCAAGTACTACGTGAACCATTCCC

CTAAGGACATTTGTACGTCAAATTCTTCAC

GTGAAGAATTTGACGTACAAATGTCCTTAG

GCTCTCAGCCGCAAGGACCGCATAC

GTATGCGGTCCTTGCGGCTGAGAGC

CCTTATATGGGTGTTGTGAAGCAGGATAGAAC

GTTCTATCCTGCTTCACAACACCCATATAAGG

GGCTCTACCTAATCATCCTCTTGACACAAC

GTTGTGTCAAGAGGATGATTAGGTAGAGCC

GACTGTGTGTGGAGCAGCTATTTGCTTCGGC GCCGAAGCAAATAGCTGCTCCACACACAGTC urea, $45 \mathrm{mM}$ Tris, $45 \mathrm{mM}$ boric acid, and 1 mM EDTA for $15 \mathrm{~min}$, then subject to blotting and Northern analysis as described above, using Ultra-Hyb Oligo hybridization buffer (Ambion) and a probe against psiRNA 7.01 .

\section{SUPPLEMENTAL DATA}

Supplemental material can be found at http://www.rnajournal.org.

\section{ACKNOWLEDGMENTS}

We thank Lindsay Jones for assistance with Northern analysis, Gerti Schut and Mike Adams (University of Georgia) for P. furiosus material and technical assistance with chromatography, and Alex Hüttenhofer (University of Innsbruck) for encouragement. This work was supported by NIH R01-GM54682 (M.P.T. and R.M.T.).

Received July 2, 2008; accepted August 21, 2008.
(20 pmol) were $5^{\prime}$ end-labeled with T4 Polynucleotide Kinase (Ambion) and $\gamma$ - $\left[{ }^{32} \mathrm{P}\right]$-ATP (specific activity $>7000 \mathrm{Ci} / \mathrm{mmol}, \mathrm{MP}$ Biomedicals) using standard protocols. Labeled probes were added to the prehybridization buffer, followed by hybridization overnight at $42^{\circ} \mathrm{C}$. Following hybridization, two washes were performed in $2 \times$ SSC, $0.5 \%$ SDS for $30 \mathrm{~min}$ at $42^{\circ} \mathrm{C}$. Resulting blots were exposed to a PhosphorImager screen for 24-72 h and scanned. The probes and sequences that were used are given in Table 2.

\section{Chromatography}

Fifteen grams of $P$. furiosus cells were lysed anerobically in $200 \mathrm{~mL}$ $50 \mathrm{mM}$ Tris ( $\mathrm{pH} \mathrm{8.0)}$ in the presence of $4 \mathrm{mg} / \mathrm{L}$ RNase-free DNase (Sigma). The extract was subject to ultracentrifugation at $113,000 \mathrm{~g}$ for $2 \mathrm{~h}$ (Optima L-90K, Beckman-Coulter). The resulting S100 extract was applied to a $60 \mathrm{~mL}$ DEAE Sepharose-FF column and eluted using a $0-500 \mathrm{mM} \mathrm{NaCl}$ gradient. The resulting fractions were analyzed by isolating RNAs from $250 \mu \mathrm{L}$ of each $30 \mathrm{~mL}$ fraction using the Trizol LS protocol (Invitrogen). The RNAs were separated on $10 \%$ polyacrylamide $7 \mathrm{M}$ urea gels, blotted, and subject to Northern analysis as described above, using the OligoUltraHyb hybridization buffer for both pre-hybridization and hybridization. See above for probe sequences.

\section{Native Northern analysis}

Forty microliters of DEAE fractions from peaks A-C were separated on a $4 \%-20 \%$ polyacrylamide gel (Bio-Rad) using SDS-free running buffer ( $25 \mathrm{mM}$ Tris, $19.2 \mathrm{mM}$ glycine). Deproteininzed samples were analyzed in parallel. Gels were run at $50 \mathrm{~V}$ for $3-4 \mathrm{~h}$ at room temperature. The gel was soaked in $5 \mathrm{M}$

\section{REFERENCES}

Aravin, A.A., Hannon, G.J., and Brennecke, J. 2007. The Piwi-piRNA pathway provides an adaptive defense in the transposon arms race. Science 318: 761-764.

Barrangou, R., Fremaux, C., Deveau, H., Richards, M., Boyaval, P., Moineau, S., Romero, D.A., and Horvath, P. 2007. CRISPR provides acquired resistance against viruses in prokaryotes. Science 315: 1709-1712.

Bolotin, A., Quinquis, B., Sorokin, A., and Ehrlich, S.D. 2005. Clustered regularly interspaced short palindrome repeats (CRISPRs) have spacers of extrachromosomal origin. Microbiology 151: 2551-2561.

Deveau, H., Barrangou, R., Garneau, J.E., Labonte, J., Fremaux, C., Boyaval, P., Romero, D.A., Horvath, P., and Moineau, S. 2008. Phage response to CRISPR-encoded resistance in Streptococcus thermophilus. J. Bacteriol. 190: 1390-1400.

Godde, J.S. and Bickerton, A. 2006. The repetitive DNA elements called CRISPRs and their associated genes: Evidence of horizontal transfer among prokaryotes. J. Mol. Evol. 62: 718-729.

Grissa, I., Vergnaud, G., and Pourcel, C. 2007. The CRISPRdb database and tools to display CRISPRs and to generate dictionaries of spacers and repeats. BMC Bioinformatics 8: 172 .

Haft, D.H., Selengut, J., Mongodin, E.F., and Nelson, K.E. 2005. A guild of 45 CRISPR-associated (Cas) protein families and multiple CRISPR/Cas subtypes exist in prokaryotic genomes. PLoS Comput. Biol. 1: e60.

Hannon, G.J. 2002. RNA interference. Nature 418: 244-251.

Hartig, J.V., Tomari, Y., and Forstemann, K. 2007. piRNAs-The ancient hunters of genome invaders. Genes \& Dev. 21: 1707-1713.

Horvath, P., Romero, D.A., Coute-Monvoisin, A.C., Richards, M., Deveau, H., Moineau, S., Boyaval, P., Fremaux, C., and Barrangou, R. 2008. Diversity, activity, and evolution of CRISPR loci in Streptococcus thermophilus. J. Bacteriol. 190: 1401-1412.

Jansen, R., Embden, J.D., Gaastra, W., and Schouls, L.M. 2002. Identification of genes that are associated with DNA repeats in prokaryotes. Mol. Microbiol. 43: 1565-1575. 
Kim, V.N. 2006. Small RNAs just got bigger: Piwi-interacting RNAs (piRNAs) in mammalian testes. Genes \& Dev. 20: 19931997.

Klein, R.J., Misulovin, Z., and Eddy, S.R. 2002. Noncoding RNA genes identified in AT-rich hyperthermophiles. Proc. Natl. Acad. Sci. 99: $7542-7547$.

Lau, N.C., Lim, L.P., Weinstein, E.G., and Bartel, D.P. 2001. An abundant class of tiny RNAs with probable regulatory roles in Caenorhabditis elegans. Science 294: 858-862.

Lillestol, R.K., Redder, P., Garrett, R.A., and Brugger, K. 2006. A putative viral defence mechanism in archaeal cells. Archaea 2: $59-72$.

Lin, H. 2007. piRNAs in the germ line. Science 316: 397.

Makarova, K.S., Aravind, L., Grishin, N.V., Rogozin, I.B., and Koonin, E.V. 2002. A DNA repair system specific for thermophilic Archaea and bacteria predicted by genomic context analysis. Nucleic Acids Res. 30: 482-496.

Makarova, K.S., Grishin, N.V., Shabalina, S.A., Wolf, Y.I., and Koonin, E.V. 2006. A putative RNA-interference-based immune system in prokaryotes: Computational analysis of the predicted enzymatic machinery, functional analogies with eukaryotic RNAi, and hypothetical mechanisms of action. Biol. Direct 1: 7.

Mojica, F.J., Diez-Villasenor, C., Garcia-Martinez, J., and Soria, E. 2005. Intervening sequences of regularly spaced prokaryotic repeats derive from foreign genetic elements. J. Mol. Evol. 60: $174-182$.

Nishida, K.M. and Siomi, M.C. 2006. Molecular mechanisms of RNA silencing by siRNA, miRNA and piRNA. Tanpakushitsu Kakusan Koso 51: 2450-2455.

Pourcel, C., Salvignol, G., and Vergnaud, G. 2005. CRISPR elements in Yersinia pestis acquire new repeats by preferential uptake of bacteriophage DNA, and provide additional tools for evolutionary studies. Microbiology 151: 653-663.

Sorek, R., Kunin, V., and Hugenholtz, P. 2008. CRISPR-a widespread system that provides acquired resistance against phages in bacteria and archaea. Nat. Rev. Microbiol. 6: 181-186.

Tang, T.H., Bachellerie, J.P., Rozhdestvensky, T., Bortolin, M.L., Huber, H., Drungowski, M., Elge, T., Brosius, J., and Huttenhofer, A. 2002. Identification of 86 candidates for small non-messenger RNAs from the archaeon Archaeoglobus fulgidus. Proc. Natl. Acad. Sci. 99: 7536-7541.

Tang, T.H., Polacek, N., Zywicki, M., Huber, H., Brugger, K., Garrett, R., Bachellerie, J.P., and Huttenhofer, A. 2005. Identification of novel non-coding RNAs as potential antisense regulators in the archaeon Sulfolobus solfataricus. Mol. Microbiol. 55: 469-481.

Tyson, G.W. and Banfield, J.F. 2008. Rapidly evolving CRISPRs implicated in acquired resistance of microorganisms to viruses. Environ. Microbiol. 10: 200-207. 

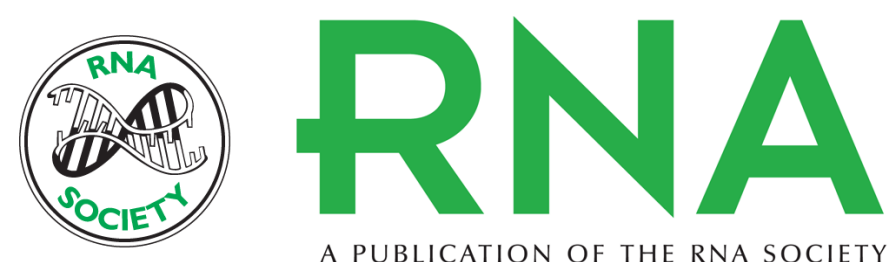

A PUBLICATION OF THE RNA SOCIETY

\section{Prokaryotic silencing (psi)RNAs in Pyrococcus furiosus}

Caryn Hale, Kyle Kleppe, Rebecca M. Terns, et al.

RNA 2008 14: 2572-2579 originally published online October 29, 2008

Access the most recent version at doi:10.1261/rna.1246808

Supplemental

Material

References

\section{License}

Email Alerting Service
http://rnajournal.cshlp.org/content/suppl/2008/10/30/rna.1246808.DC1

This article cites 25 articles, 10 of which can be accessed free at: http://rnajournal.cshlp.org/content/14/12/2572.full.html\#ref-list-1 